\title{
Evaluation of Cancer Progression Using Dynamic Entropy Changes and Thermography
}

\author{
Mohammad Hassan Khoshgoftar Manesh ${ }^{1,2 *}$, Ali Akbar Rezazadeh ${ }^{1,2}$, Tayebeh. Modaresi Movahed ${ }^{2,3}$ Hamid \\ Reza Mirzaei ${ }^{4}$ \\ ${ }^{1}$ Energy, Environment and Biologic Research Lab (EEBRlab), Division of Thermal Sciences \& Energy Systems, \\ Department of Mechanical Engineering, University of Qom, Qom, Iran \\ ${ }^{2}$ Center of Environmental Research, University of Qom, Qom, Iran \\ ${ }^{3}$ Department of Electrical Engineering, University of Qom, Qom, Iran \\ ${ }^{4}$ Department of Medical Immunology, School of Medicine, Tehran University of Medical Sciences, Tehran, Iran \\ E-mail: m.khoshgoftar@qom.ac.ir ; mh.khoshgoftar@gmail.com
}

Received 23 February 2021, Revised 18 March 2021, Accepted 11 April 2021

\begin{abstract}
Entropy is producing during any irreversible process. In the cancer cells, the entropy generation measures the irreversibility; so, the cancer cells have higher entropy generation than the healthy cells. The entropy generation rate shows the amount of robustness, progression, and invasion of the cancer cells. From a thermodynamic aspect, cancer's origin and growth is an irreversible process, and the thermodynamic variables such as the cell volume, temperature, and entropy will change during this process. In this paper, a procedure based on experimental data is proposed to calculate dynamic entropy generation in the tumoral tissues by dynamic thermography and measurement of tumor size. The dynamic changes in the volume, temperature, and entropy associated with tumor cells over time are tested and evaluated in this regard. An in vivo assay has been developed to measure and analyze these changes. This assay investigated the growth of 4T1 Breast Tumor in $55 \mathrm{BALB} / \mathrm{c}$ mice over time. Infrared thermography has been employed to evaluate dynamic temperature changes of the tumors. The computer code has been developed to gather important data from tumoral and healthy mice's images to compute considered temperature differences and entropy generation associated with tumoral tissues. To better evaluate tumor tissue, the Micro PET Images are used to verify volume changes of tumors. The relation between the volume and temperature gradient of tumor cells has detected by measuring during the experiment. The entropy of tumor cells was studying and calculating during the process of tumor changes. Results show that entropy generation as the main concept of thermodynamic is a strong tool for the analysis of cancer cells and has a strong relationship with cancer growth.
\end{abstract}

Keywords: Breast Cancer, Entropy, Volume, Temperature, Infrared Thermography, Micro PET.

\section{Introduction}

Cancer refers to a disease in which the cells have lost their specialization and control over normal growth. According to reports from the WHO (World Health Organization), Cancer is the second major cause of death in the world and is responsible for an evaluated 9.6 million deaths in 2018 [1].

The living cell can be described as a thermodynamic system whose structure and behavior result from processes and thermodynamic reactions within the cell and among the cell and its environment.

Despite many studies and research in this field, cancer facts are still obscure, and numerous therapies such as surgery, radiotherapy, and chemotherapy have not been quite successful. Applying timely diagnosis and effective treatment can increase the chance for recovery in patients. For cancer diagnosis, some enhanced techniques are available such as CT, MRI, and PET scanning. However, they are expensive and not flexible, and widely accessible. Widely available, quick, and accurate tools for objective measurement are needed for diagnosing cancer and examining its changes over time [2].
Thermal imaging can be an excellent tool that is an accurate, non-contact, non-invasive, and real-time measurement related to the temperature of the surface.

Thermal imaging technology has been made and developed in clinical medicine since the 1960s and was initially well approved and accepted [1-4]. Despite that, after 1977, the medical community has lost interest in this method of diagnosis. This diagnostic method's failure has been described in the interpretation of thermal images and the use of non-digital images $[1,4]$. The thermography concept is based on the detection and visualization of varying heat emitted by the body, and it has been appending to the procedures available for inspection and testing for breast cancer and the further investigation and studies of the action of these lesions. Thermal images can also recognize the actual size of the thermal modifications associated with foci with precise calibration [2]. It is an evident and strong reason that thermography is a very promising technique for testing and regenerating patients for breast cancer [7].

Ma et al. estimated the temperature of the tumor in nanoparticle magnetic hyperthermia using thermography [8]. Regression functions are made to 
determine the relationship, which is useful for the fast tumor's inner temperature estimation using infrared thermography.

In the past years, there has been extensive research that has determined thermography to have the ability to find and identify breast abnormalities that other recognition approaches may not have detected. Although no single device supplies supreme foreseeability, a combination that merges thermography, such as mammography, may promote both particularity and sensitivity [9]. It was indicated that using thermography alone had a sensitivity of $83 \%$ in revealing breast cancer, while the integration of thermography and mammography had a $95 \%$ sensitivity [4]. There is no detection device currently available that supplies $100 \%$ predictability of the existence of a cancerous tumor. A biopsy is the only deterministic diagnostic approach is [4].

When signs or symptoms of breast disease exist, a complete test by clinical test, biopsy, thermography, and mammography is vital. Thermography may be applied to achieve further data by recognizing metastatic lesions in superficial lymph nodes or bones [5].

Jo and Kim used infrared thermography as a diagnostic tool to evaluate abdominal skin temperature between fertile and infertile women. They indicated that infertility was related to lower AT [10].

Bousselham et al. used the thermography method on GPU to demonstrate tumor position and parameter prediction. The genetic algorithm was applied to the computation of the inverse problem [11]. The equation of Pennes for bioheat was solved through GPU parallel finite difference method.

The hot spot over a tumor and its high thermal gradient are the most major factors to distinguish malignancy from a benign state. Increasing $1-2{ }^{\circ} \mathrm{C}$ of the skin surface temperature is usually seen at the periphery of human breast tumors. This modification is because of the blood lipids and increasing the blood flow due to tumor-related angiogenesis [6]. Thermography does not supply data on the breast's morphological specifications, but it supplies functional data on vascular and thermal tissue conditions. These functional variations are hypothesized to modify before the onset of structural variations in a diseased or cancerous state [7]. It is wellknown that physiological modifications in tissue before pathological changes [12] and investigations promote the significant role of thermography in the early detection of breast abnormalities that may be reached to cancer [7].

Das and Mishra performed a numerical study of malignant tumors in the breast of humans to predict the size, radial, and angular positions of a simultaneously. Inverse methods were carried out by a curve fitting approach [13]. Also, the result of the error of measurement in temperature was considered.

Patil and Maniyeri investigated bio-heat transfer in the breast cyst of human-based on the Finite difference method [14]. The location of the cyst has been investigated, and the simulation of multiple cysts has been done. Results are effective for the detection of cancer in the early stage of progression. Etehadtavakol and $\mathrm{Ng}$ [15] have investigated modeling bioheat transfer based on numerical approaches for exact measurements of the skin surface. Thermal distribution for living tissues has been modeled based on Pennes bioheat. A precise mathematical model for heat transfer was essential for thermography.

The previous studies indicate that it is always possible to gain tumor parameters with precise static or transient measurement information. Nerveless, for noisy temperature information, the application of a transient approach demonstrated an advantage over the steadystate condition, which failed due to the very small temperature differences between the tumoral skin and healthy skin.

In this study, we have analyzed the dynamics of the cancer system by using thermodynamic variables. For this purpose, we have investigated the changes in the temperature gradient and volume of $4 \mathrm{~T} 1$ Breast Tumor in $53 \mathrm{BALB} / \mathrm{c}$ mice over time. We have used thermal imaging to study the dynamic changes in tumor temperature gradient and compare these changes to tumor volume changes. With a proven nonlinear relation and the volume and temperature gradient values, we have calculated the entropy generation values for tumor cells. In the new researches, entropy has been suggested as a strong tool for studying cancer behavior and its stationary states.

\section{Thermodynamic Fundamentals}

From a thermodynamic aspect, cancer can be considered as an open [8] and nonlinear dynamical system [17] in the non-equilibrium thermodynamic state [9], which is selforganized in space and time [10], containing high complexity [11], robustness [12] and adaptability [13]. Irreversibility is the fundamental definition and measurement for evaluating the cycles and systems [23-26]. Cancer's appearance and progression is an irreversible process, and entropy generation measures the cancer system behavior [19]. Entropy is a state function based on the system equilibrium, and we can only calculate the entropy differences [21] because, in reality, nothing is generated $[24,27]$. Considering the second law of thermodynamic the total entropy of systems is composed of two sections: the entropy change that will be achieved conversion overall the system borders as $\Delta S$ and the entropy generation expressed as the entropy change the consequences of the irreversibility as $S_{g}$ and it is always $S_{g} \geq 0$ [27].

$s=\int\left(\frac{\partial Q}{T}\right)=S_{g}+\Delta S$

The total entropy of the system can be calculated as a relation (1). In this relation, $Q$ is the heat transfer, $T$ the temperature of the thermostats.

In other words, entropy is a measure of disorder, and life should remain in a low-entropy state [27]. Generating large amounts of entropy in the industrial systems reduces these systems' efficiency and in the biological systems is a sign of dysfunction and disease. Typically, the entropy generation rate of cancer cells is always higher than healthy cells (about two times) when no external forces are used [30]. The entropy generation rate is the characteristic behavior of the tumor and indicates its progression, invasion [31], and robustness [32], so entropy can be a useful and effective tool for diagnosing cancer cells from healthy cells and measuring its progression, invasion, and robustness. It's has been proven that the entropy of living cells can be calculated in terms of two variables: the volume and temperature of cells. Studies have shown that these two 
thermodynamic variables are important independently in detecting cancer and analyzing cancer cell behavior. As mentioned in the previous section, the temperature has been regarded as one of the main parameters in the analysis of cancer advances [33], and recently, by using thermal imaging, the temperature has been studied as an interesting topic of research concerning the behavior of tumoral and normal cells [34]. Umberto Lucia [18] has calculated a critical value for the volume of cancer tumors based on entropy generation and the values of initial tumor volume and the temperature gradient between tumoral and healthy cells. This critical volume is the beginning of metastasis. Therefore entropy, volume, and temperature of tumor cells are three important factors for studying cancer and predicting its behavior. The cancer system can grow only for values of thermodynamic quantities in a stationery range, and outside of this range, it cannot develope $[18,30]$. It has been mentioned that the entropy production rate, $S_{g}$ contains five terms $[14,19,35,36]$ :

1. The entropy generation because of the thermal flux obtained by a temperature difference; can be calculated as:

$$
S_{g . t f}=\alpha \Delta T L^{2} \text { with } \alpha=\frac{3.3 \times 10^{12} \tau_{1}}{T}
$$

Where $L$ is the typical length of a cell, $\Delta T[\mathrm{~K}]$ is the temperature difference in $L, \tau_{1}[\mathrm{~s}]$ is the time associated with the heat flow obtained by temperature difference and $T$ $[\mathrm{K}]$ is the temperature. The temperature of normal cells is homogeneous and $\Delta \mathrm{T}=0$; therefore, there is no entropy generation in consequence of heat flow. Nevertheless, an increase in temperature for some cancers has been reported. We have considered $\mathrm{T}=305 \mathrm{~K}$ based on laboratory conditions and $\tau_{1}=15 \mathrm{~ms}$

2. The entropy generation because of the diffusion current achieved by a potential chemical gradient;

$S_{g . a k}=\beta L^{3}$ with $\beta=\frac{3 \times 10^{7} \tau_{2}}{T}$

In which $\tau_{2}$ is the process lifetime. The chemical potential gradient usually occurs in the cell cytoplasm. This work $\tau_{2}$ has been considered $10 \mathrm{~ms}$.

3. The entropy generation in consequence of the velocity gradient integrated with viscous stress;

$S_{g . v g}=\frac{\gamma}{L^{2}}$ with $\gamma=\frac{4.7 \times 10^{12} \tau_{3}}{T}$

In which $\tau_{3}$ is the process lifetime. Due to the low amount of entropy generation in this term $S_{g, v g}$ has been ignored and $\tau_{3}$ has not been calculated.

4. The entropy generation because of the chemical reaction rate obtained by a Gibbs energy decrease (affinity);

$S_{(\text {g.cr })}=\varepsilon L^{3}$ with $\varepsilon=0.523 \tau_{4} \sum N_{i} \frac{A_{i}}{T}$

In which $\tau_{4}$ is the time associated with the rate of chemical reaction obtained by affinity, $N$ is the number per unit time, and chemical reaction related to its volume. $A$ is the affinity, examined as the changes of the standard Gibbs free energy. In this work, we have considered $\tau_{4}=17 \mathrm{~ns}$ and the value of the term $\sum N_{i} \frac{A_{i}}{T}$; due to the cell proliferation equal to $10^{16}$.

5. The entropy generation because of the dissipation of the work completed by an external force field. It is not possible to analyze an overall relation for this term for a cell due to its associates with the cell's environment.

So, the entropy generation for a cell in the case where no external energy is applied can be calculated as [23]:

$$
\begin{aligned}
& S_{g}=S_{g . t f}+S_{g . a k}+S_{g . v g}+S_{g . c r}= \\
& -(\alpha+\gamma) \frac{1}{T}-\frac{\alpha T_{0}}{T^{2}}-(\beta+\varepsilon) \frac{v}{T}=\alpha \Delta T L^{2}+\beta L^{3}+\frac{\gamma}{L^{2}}+\varepsilon L
\end{aligned}
$$

This relation is only based on the values of volume and temperature gradient and with $\alpha, \beta, \gamma$, and $\varepsilon$ are constant in the defined environment. It must be underlined that this relation can be used for the entire tumor cells.

\section{Material and Method}

\subsection{Experimental Method}

In this study, assuming cancer as a thermodynamic system, the dynamic changes of the thermodynamic variables such as the volume, temperature gradient, and entropy of the tumor system have been investigated during the appearance and progression of cancer. It was necessary to inject cancer cells into some mice because of analyzing the changes in these thermodynamic variables and their relationship to different cancer stages. The thermodynamic behavior of the tumor cells has been studied from the time of injection. The volume and temperature of the tumor were measured every day. For this purpose, $62 \mathrm{BALB} / \mathrm{c}$ mice have been selected. The 4T1 cancer cells were injected Subcutaneously into the breast tissue of 55 mice, and the other was kept healthy as the control group. The temperature changes in the breast tissue and the changes in tumor volume were monitored daily from tumor cell injection. The volume of the tumor measured using a laboratory digital caliper, and an IR camera measured the tumor's temperature. Using the relation (6), the entropy of the tumors was calculated by using the volume and temperature gradient of the tumor cells. All of the mice were kept in similar conditions and all of the measurements were done while the mice were awake and without any sedation.

Figure 1 shows the BALB/c mice and the camera test to taking thermography before the injection of tumoral cells.
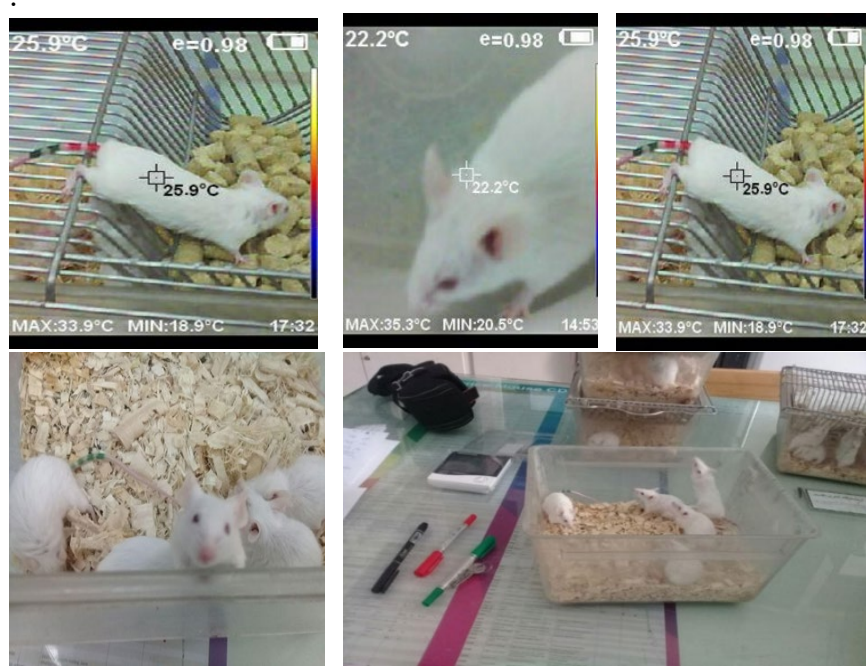

Figure 1. The BALB/c mice and the camera test to taking thermography before the injection of tumoral cells.

\subsection{Model of 4T1 Breast Tumor for Mouse}

The 4T1 mammary carcinoma is chosen for experiments. The 4T1 is an appropriate experimental animal model for human mammary cancer. The 4T1 tumor 
is extremely aggressive and tumorigenic. Unlike most tumor models, it can spontaneously metastasize from the initial tumor in the mammary gland to multiple distant positions, including blood, lung, lymph nodes, brain, liver, and bone [37]. As shown in Figure 2, the selected cases injected 700,000 4T1 cancer cells subcutaneously based on [37] procedure.

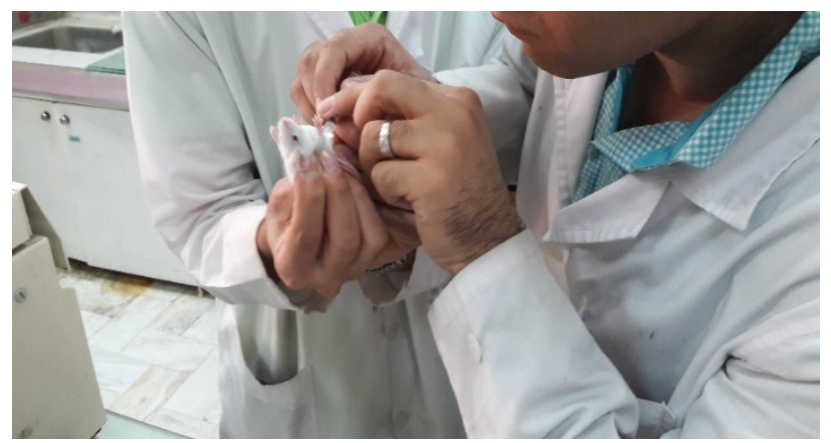

Figure 2. The demonstration of the tumor injection into the $B A L B / c$ mice.

\subsection{The Volume measurement}

After the injection, the tumors were grown as volumetric shapes that were approximately sphere or elliptical. The tumor sizes were measured with a digital caliper, and based on the geometric shape, the volume was calculated.

Most measurements of the tumor are performed manually by mechanical calipers, which have low precision and weak reproducibility. The measurement is performed by compressing the tumor between the caliper blades. The size of the tumor is then determined from the caliper. Also, to the inherent mistakes defined by this process, there is relevancy that the attendant mechanical disruption may overset tumor progress [6].

\subsection{The temperature measurement}

A Hti Model HT-18 thermal camera was used to examine the surface temperature of the tumoral tissues. Its accuracy is $\pm 2{ }^{\circ} \mathrm{C}$, thermal sensitivity is $0.08{ }^{\circ} \mathrm{C}$, and resolution is $220 \times 160$. All of the measurements were considered in a control room, and the camera was calibrated. The temperature variations were measured at the tumor site center. The consequence of the tumor presence in each mouse on the temperature of the surface body was predicted as the temperature gradient by the formula:

$\operatorname{TISTC}\left({ }^{\circ} \mathrm{C}\right)=\operatorname{TTC}\left({ }^{\circ} \mathrm{C}\right)-\operatorname{MTTC}\left({ }^{\circ} \mathrm{C}\right)$

Which TISTC is T u mor-Induced Surfa ce Tempe rature Change $\left({ }^{\circ} \mathrm{C}\right)$; TTC $\dot{\$}$ Tem perature of Tum or Cen ter and MTTC Mean Temp erature of T umo r cent er same area in each mouse before tumor cell injection $[6,7]$.

Against the temperature of the body surface or skin increases relevant to breast cancer, a temperature reduction occurred in the tumor site. This temperature reduction continued during the 4T1 tumor growth progressively as the volume of the tumor became larger. The interpretation for this consequence is unclear, but it may be due to the poorly vascularized nature of rapidly growing tumors [36-37]. There were no temperature changes in the breast tissues in the control group that did not receive tumor cells.

\subsection{Micro Pet Imaging}

PET is a nuclear medicine imaging technique that generates an image of the target of body tissues in the form of three-dimensional. PET scans use a small number of radioactive drugs or trackers to differentiate between healthy and unhealthy tissues. The most common radiopharmaceutical for this type of imaging (FDG) is called fluorodeoxyglucose, so sometimes this type of scan is called FDG-PET. Before the PET scan, a small FDG is injected into the animal because the cancer cells grow rapidly. In this study, $250 \mathrm{mCi}$ to $300 \mathrm{mCi}$ of $\mathrm{FDG}$ into each mouse through a vein has been injected and after one hour of injection of $0.1 \mathrm{ccs}$ of anesthetic subcutaneously. It grows more than healthy tissue, cancer cells absorb more FDG than healthy tissue, so in the CT scan images, the cancer cells are more colorful than healthy cells. The micro pet imaging is performed in the Xtrim preclinical Tehran University of Medical Sciences

The preclinical Xtrim Micro Pet scanner with highresolution (PNP Co, Tehran, Iran) includes ten detectors considering crystals of cerium-doped lutetium-yttrium oxyorthosilicate (LYSO: Ce) configured in the polygonal full-ring topology. Each block of the sensor consists of of $24 \times 24$ array integrated into a $12 \times 12 \mathrm{SiPM}$. The detector size is $52 \times 52 \mathrm{~mm}^{2}$ with an active area of $50.3 \times 50.3 \mathrm{~mm}^{2}$. Each block includes of $24 \times 24$ LYSO: Ce crystal with a pixel size of $2 \times 2 \times 10 \mathrm{~mm}^{3}$. The pixels are involved with $0.5 \mathrm{~m}$ BaSO4 reflectors. The system applied SensL ARRAYS-30035-144P.

Figure 3 a demonstrates the injection of FDG to BALB/c mice before PET imaging. Also, Figure $3 \mathrm{~b}$ shows the Micro PET imaging device's schematic that is applied in this investigation.

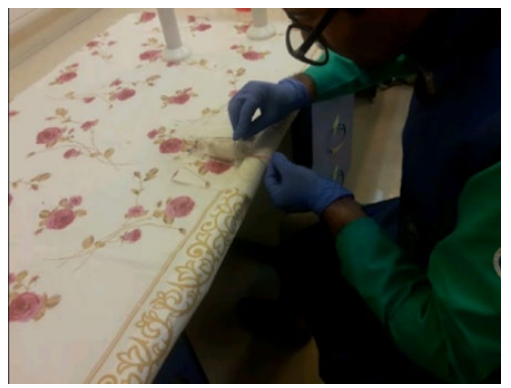

(a)

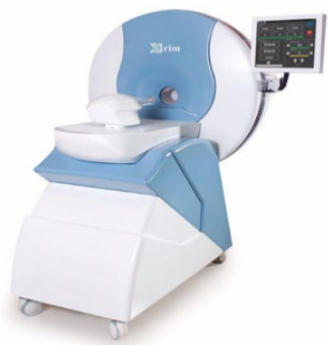

(b)
Figure 3. (a) FDG injection into the BALB/c mice (b) Micro PET Imaging Device.

\subsection{Micro Pet Imaging}

Micro Positron emission tomography (PET) imaging is a non-invasive approach to visualize and quantify the tumor microenvironment [39-40]. Micro Pet imaging is used to better visualize the size, position of the tumor.

In this study, the tumor is first injected into mice. After a few days, the effect of the tumor on the body of the mouse appears. Using thermal imaging, the condition of each mouse at the tumor injection site is checked every 4 hours and the relevant images are saved for further analysis. After creating a palpable tumor, the tumor size is recorded using a digital clipper, and the tumor volume is calculated according to the relevant geometric shape. 


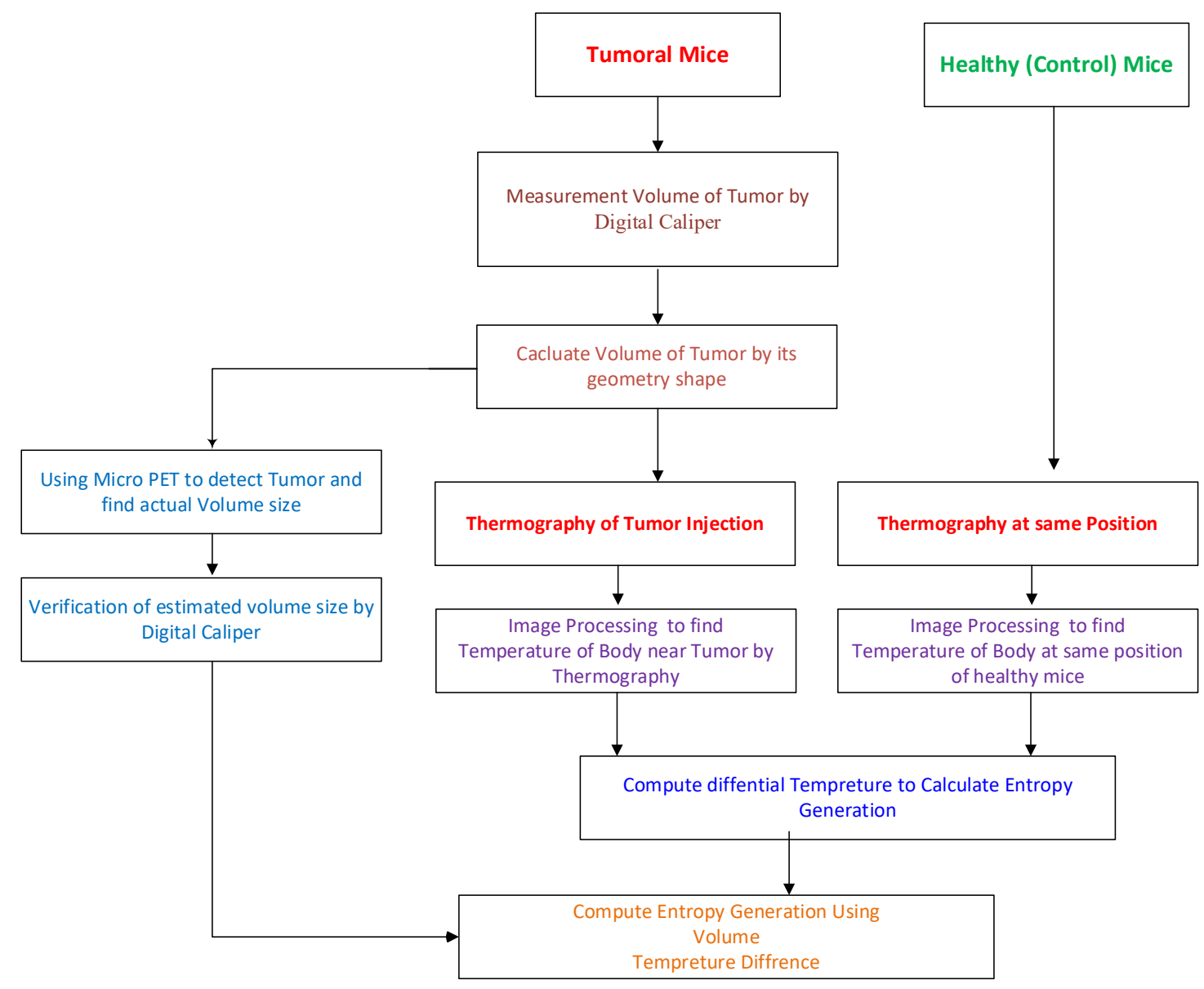

Figure 4. Proposed procedure to calculate the dynamic entropy generation in tumoral tissue.

Due to the greater accuracy in calculating tumor volume from 5 tumor mice and one healthy mouse, Micro PET images were taken, and the exact tumor volume was calculated using image processing and compared with the computed samples with a micrometer.

Also, micro PET images can be effective in analyzing the thermography results due to considering the metabolism within the tissue.

Then, the images taken from the tumor mice in the tumor area are compared with the photos taken from the healthy mice simultaneously and the same tumor location of the tumor mice in terms of temperature. This operation is done with the help of the code prepared in the content software.

The amount of entropy production is calculated using the mentioned relationships using the volume and temperature difference of the tumor tissue.

The proposed procedure to calculate dynamic entropy generation in tumoral tissue is demonstrated in Figure 4.

\section{Results}

During the initial days after injection (at least two days), there was no visible sign of any skin temperature variation in the breast tissue and a volumetric tumor. Temperature changes around the injection site were started about 2-6 days after injection and before any visible or palpable signs of a volumetric tumor. The volumetric tumor appeared after 5-10 days after injection and about 2-5 days after the start of the temperature gradient. The tumors continued to grow, and the temperature kept decreasing at the injection site.

The infrared images of healthy mice are shown in Figure 5. As illustrated, there are no temperature changes associated with tumors in breast tissue. Also, Figure 6 demonstrates the infrared images of the tumoral mice. The brown spots in the two mice above and the lower-left mouse are due to a tumor's presence. Figure 7 and 8 shows the infrared images of the Rbb case and RR case as two selected samples of tumoral mice.
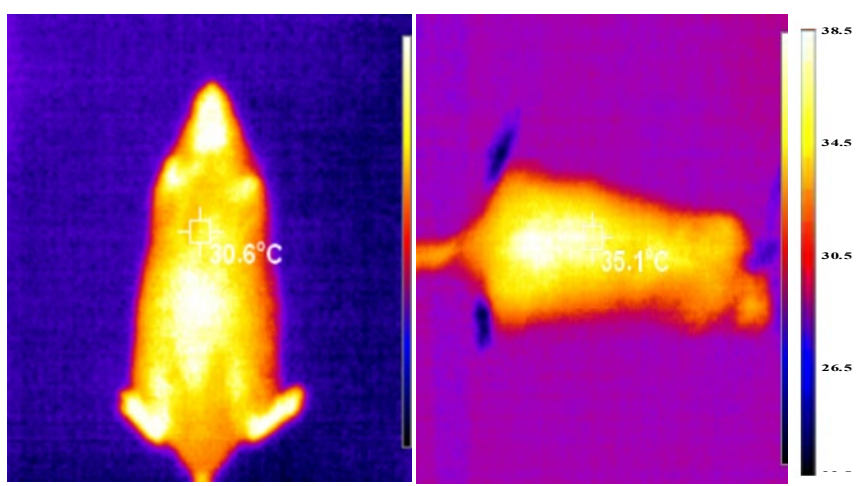

Figure 5. The infrared images of the healthy mice. 


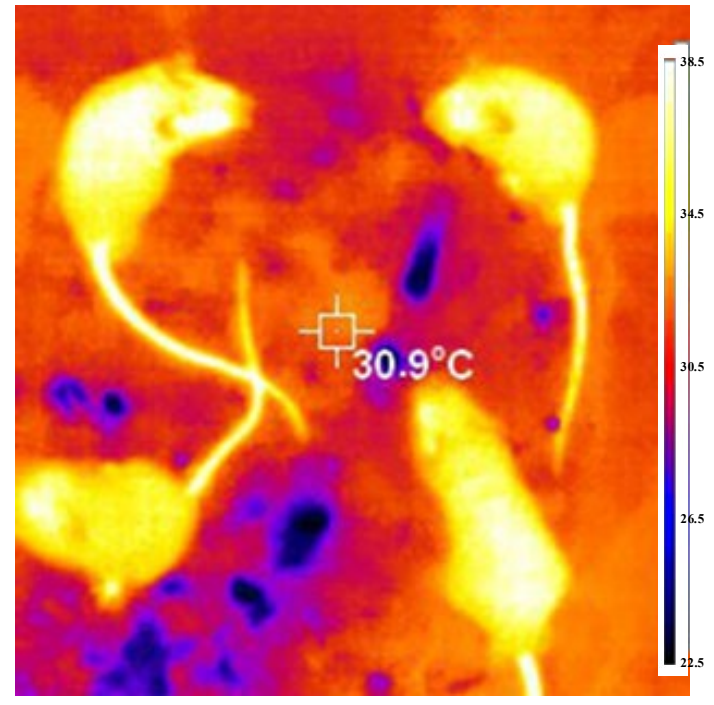

Figure 6. The infrared images of the tumoral mice.
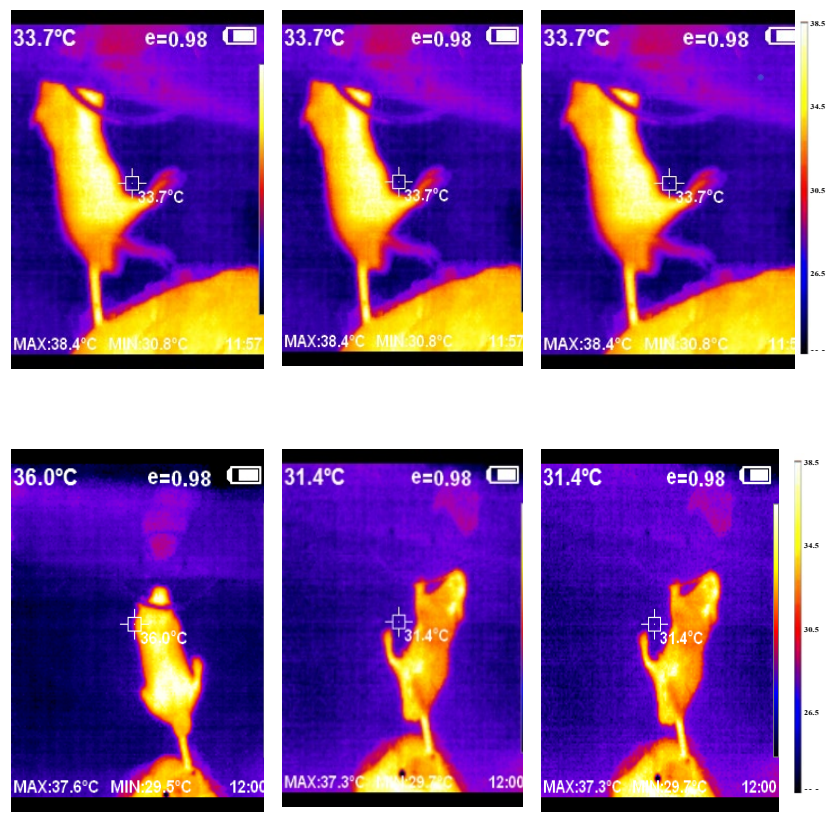

Figure 7. The infrared images of the tumoral mice-Rbb case.
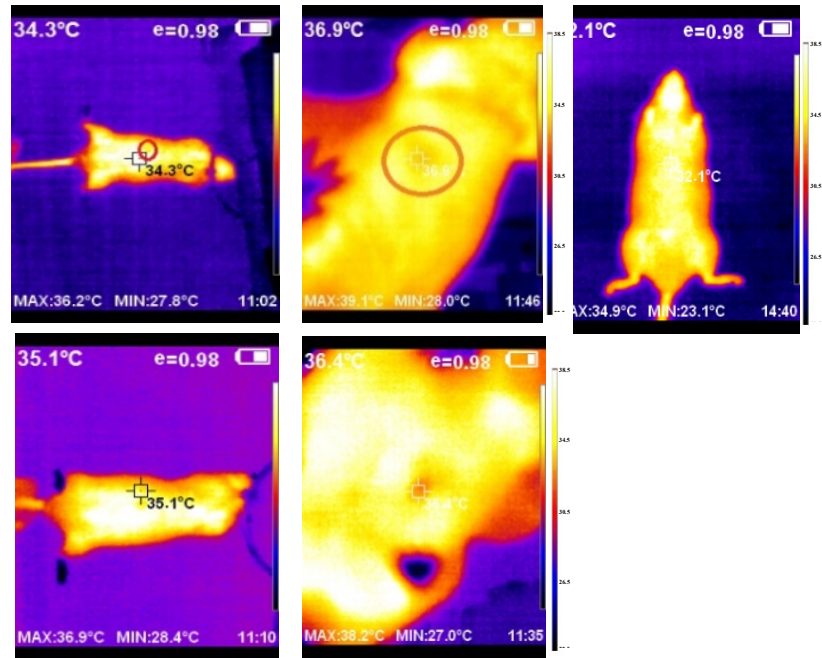

Figure 8. The infrared images of the tumoral mice-rr case.
In addition, Micro PET images of one healthy (B) mice are shown in Figure 7 over time. Furthermore, the Micro PET Images of tumoral (BG) and (BBBG) mice during the time are demonstrated in Figure 8.a and 8.b subsequently.

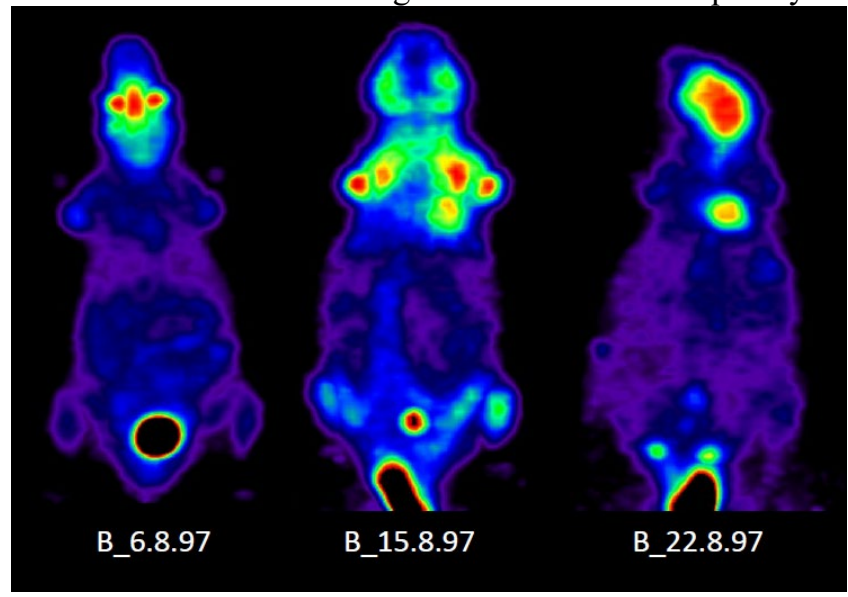

Figure 9. Results of Micro PET Images of healthy mice (B) during the time.

Micro PET images' results indicate that the computational error in calculating the volume with a digital caliper is less than two percent compared to the results obtained by the Micro PET Images.

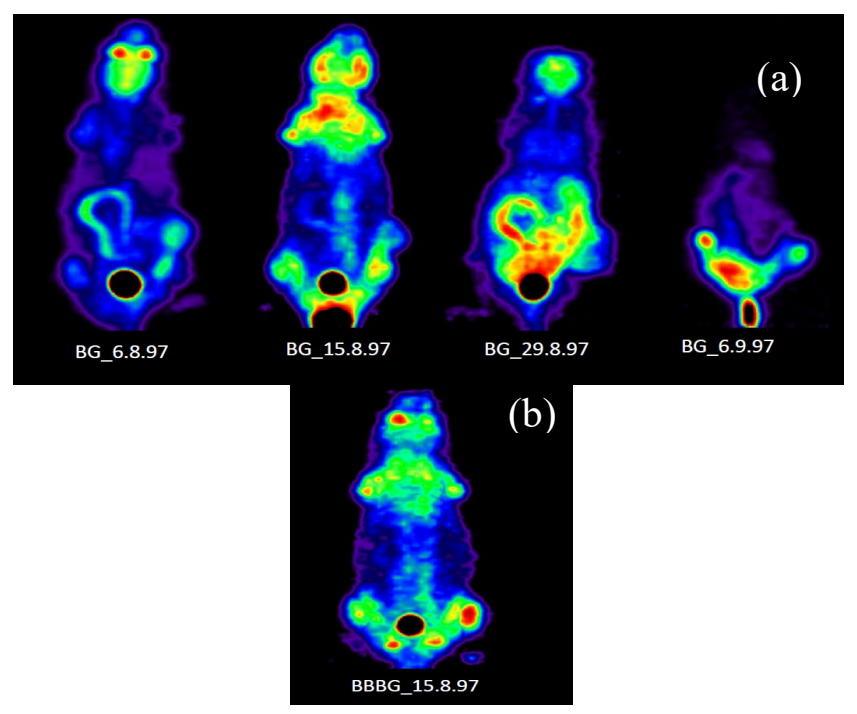

Figure 10. Results of Micro PET Images of tumoral (BG) and $(B B B G)$ mice during the time.

The magnitude of the volume changes and temperature gradient were different in mice. After about 12-26 days, 14 mice started to recover, and their tumor became smaller. As the tumors were becoming smaller in this group, the temperature continued to increase, and the temperature gradient decrease till the tumors was eliminated. The reason for the recovery of these mice was probably the resistance of their genes against this type of cancer. In Figures 11-13, the curves show the appearance and growth of tumors, then the recovery process in three mice. In the other mice, the tumor kept growing, and in most of them, cancer generated metastasis. Finally, all of them died. In Figures 14-17, the curves show cancer's appearance and progression until the death time in 6 mice for this group. 

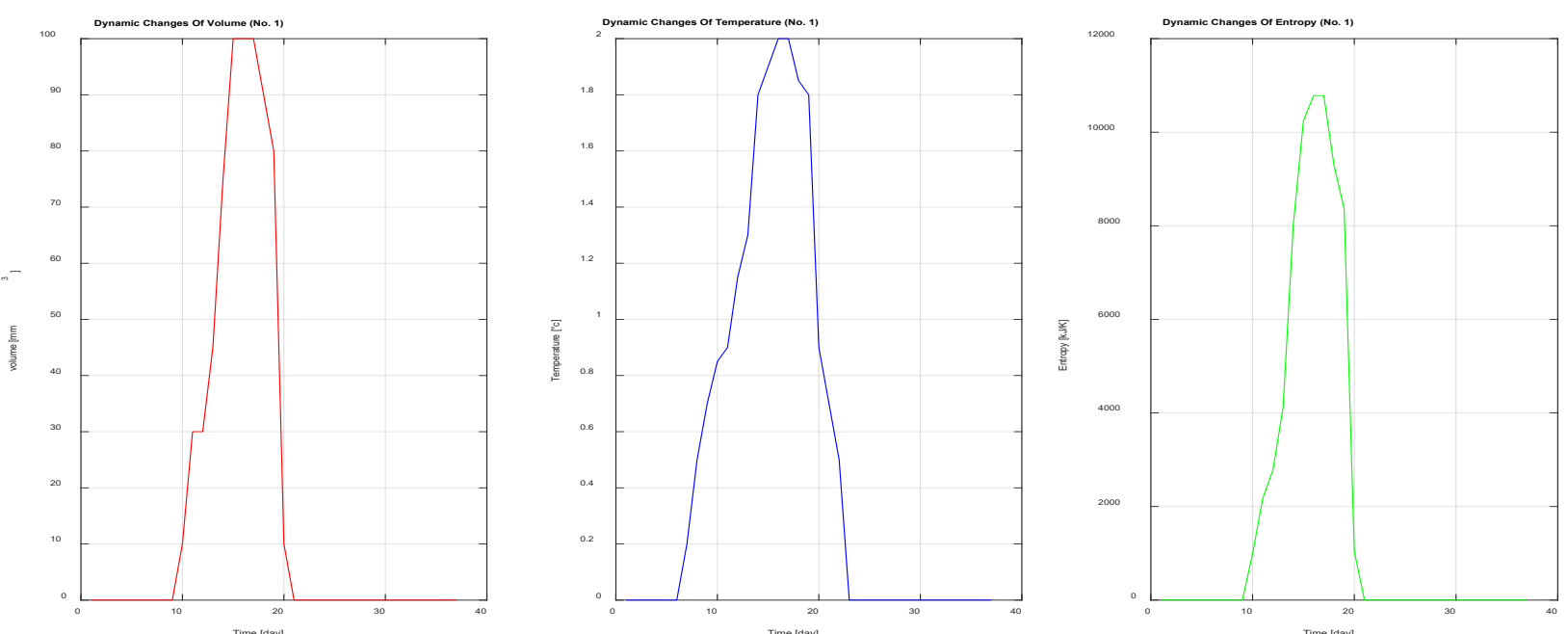

Figure 11. Dynamic changes of volume, temperature gradient, and entropy (recovered mouse No.1).
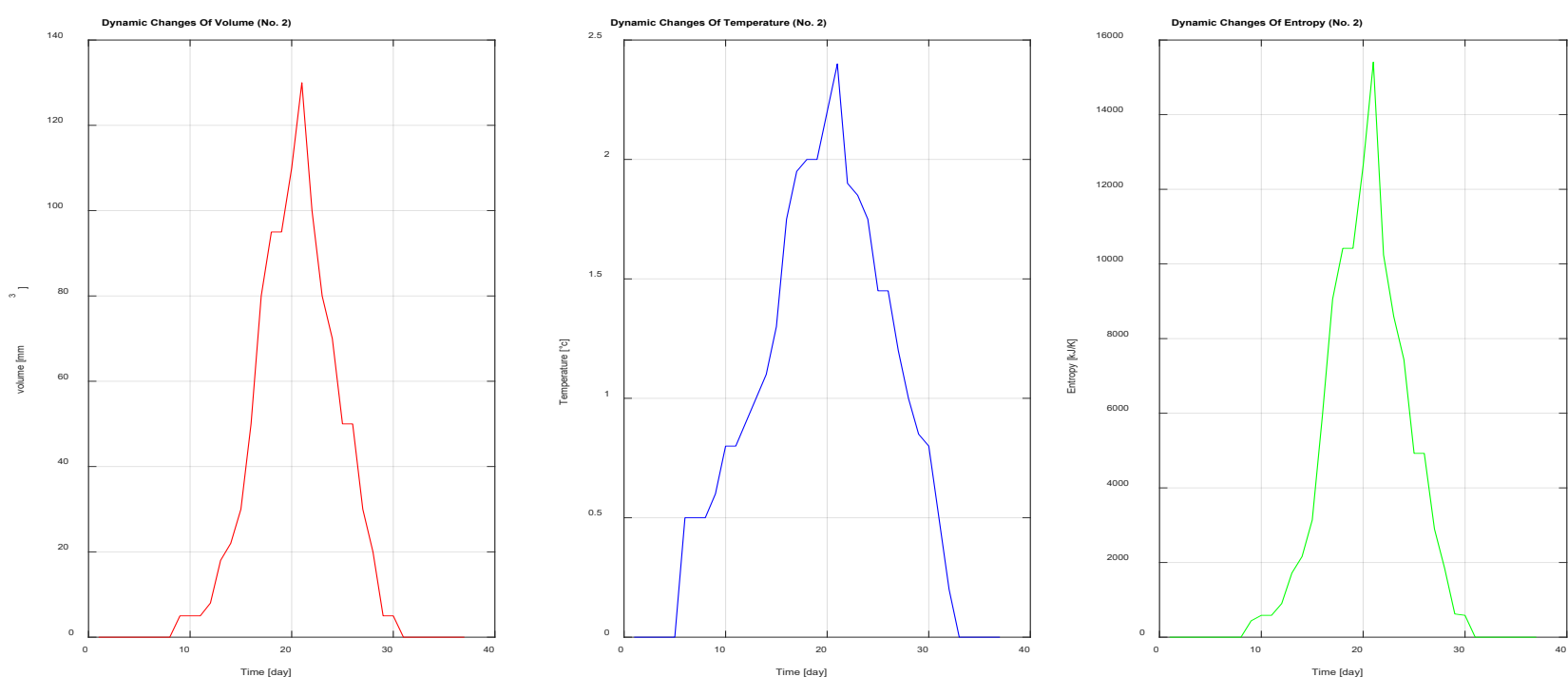

Figure 12. Dynamic changes of volume, temperature gradient, and entropy (recovered mouse No.2).
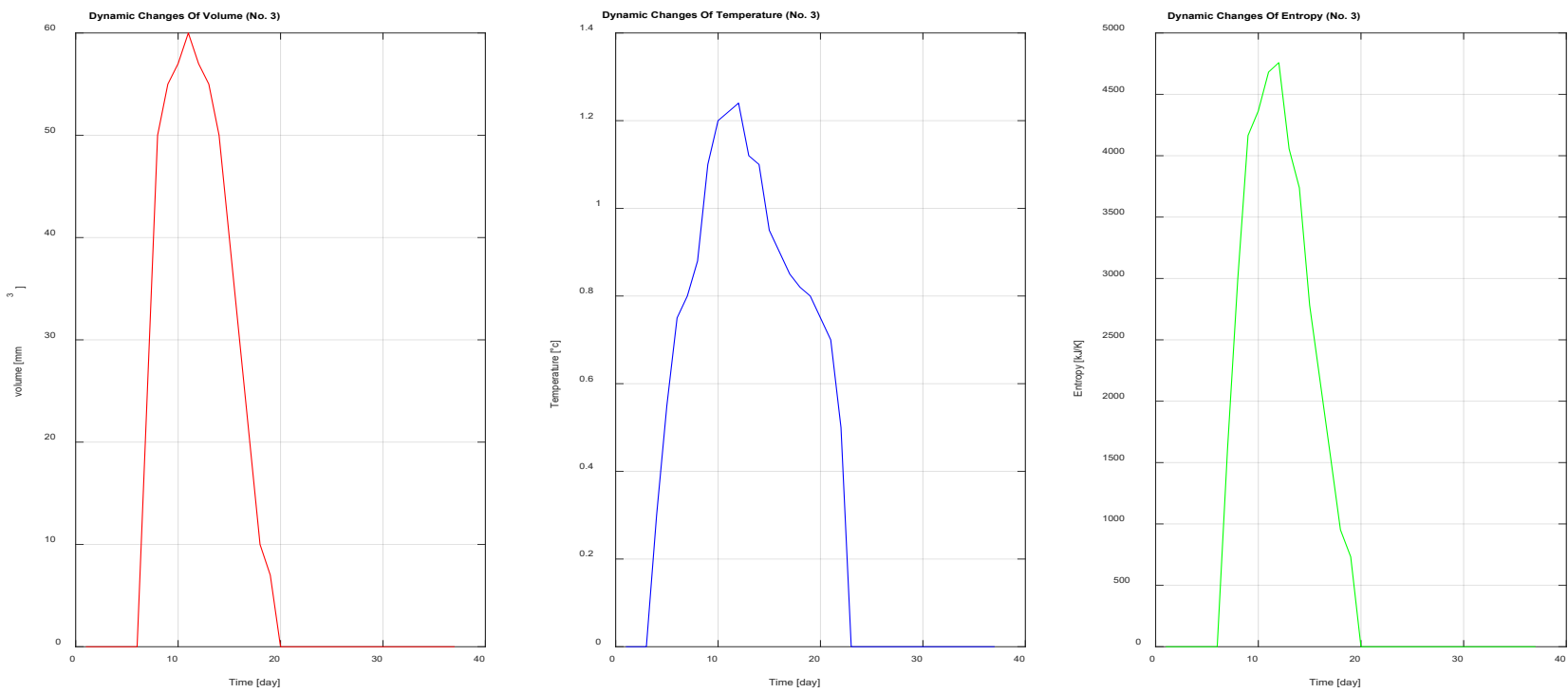

Figure 13. Dynamic changes of volume, temperature gradient, and entropy (recovered mouse No.3). 

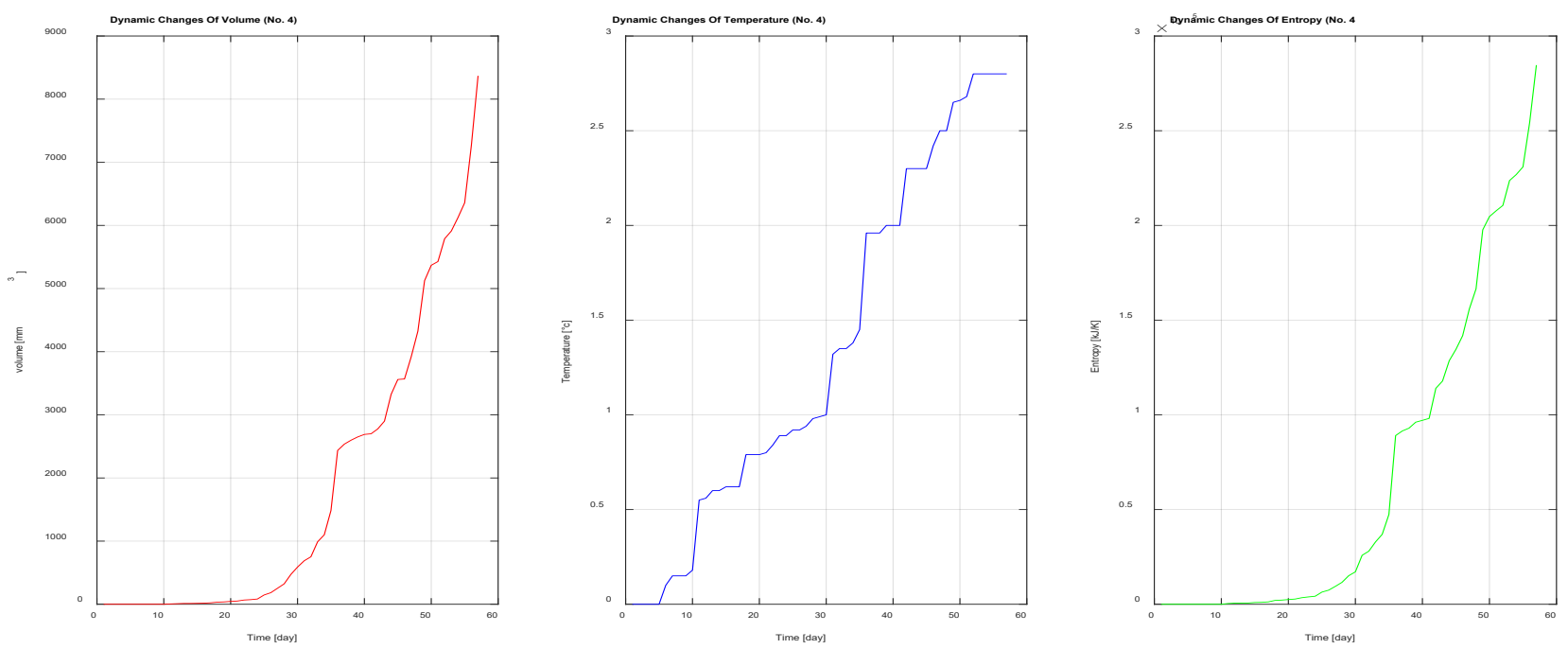

Figure 14. Dynamic changes of volume, temperature gradient, and entropy (dead mouse No.1).
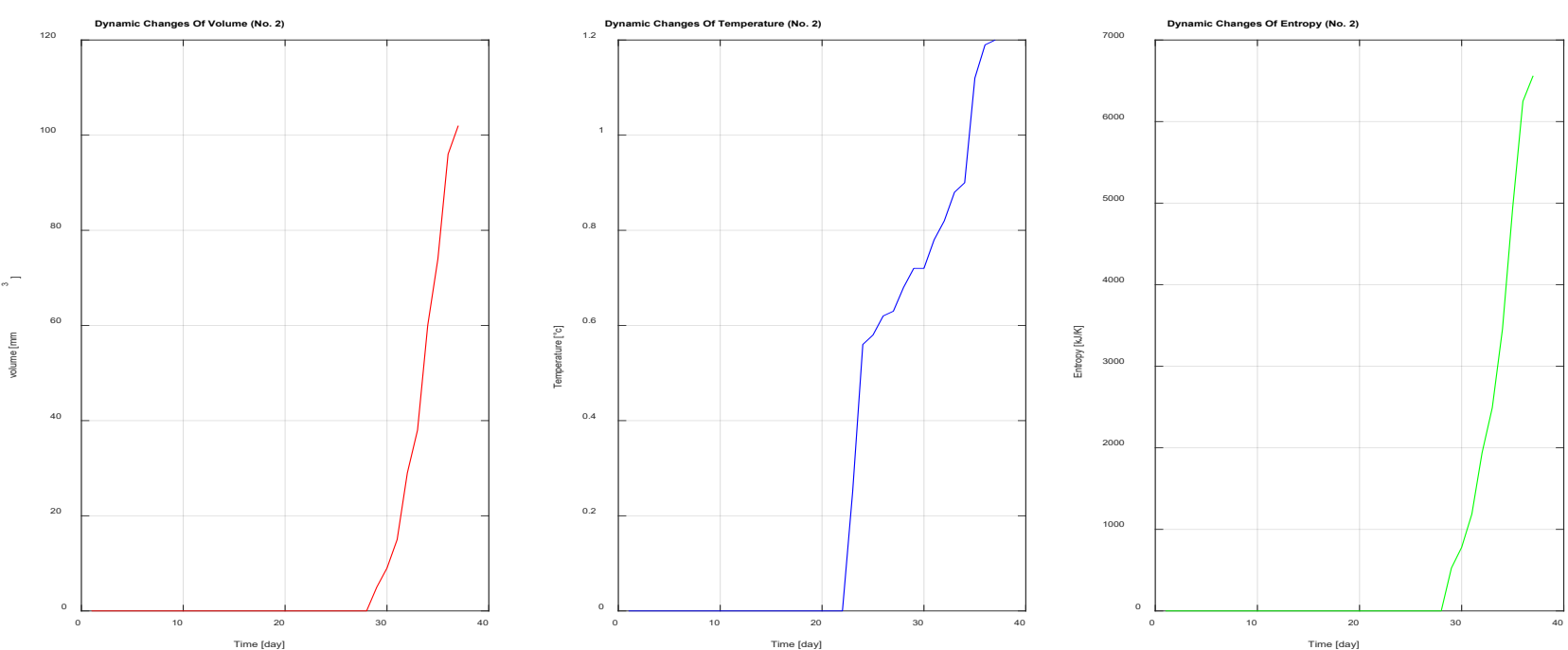

Figure 15. Dynamic changes of volume, temperature gradient, and entropy (dead mouse No.2).
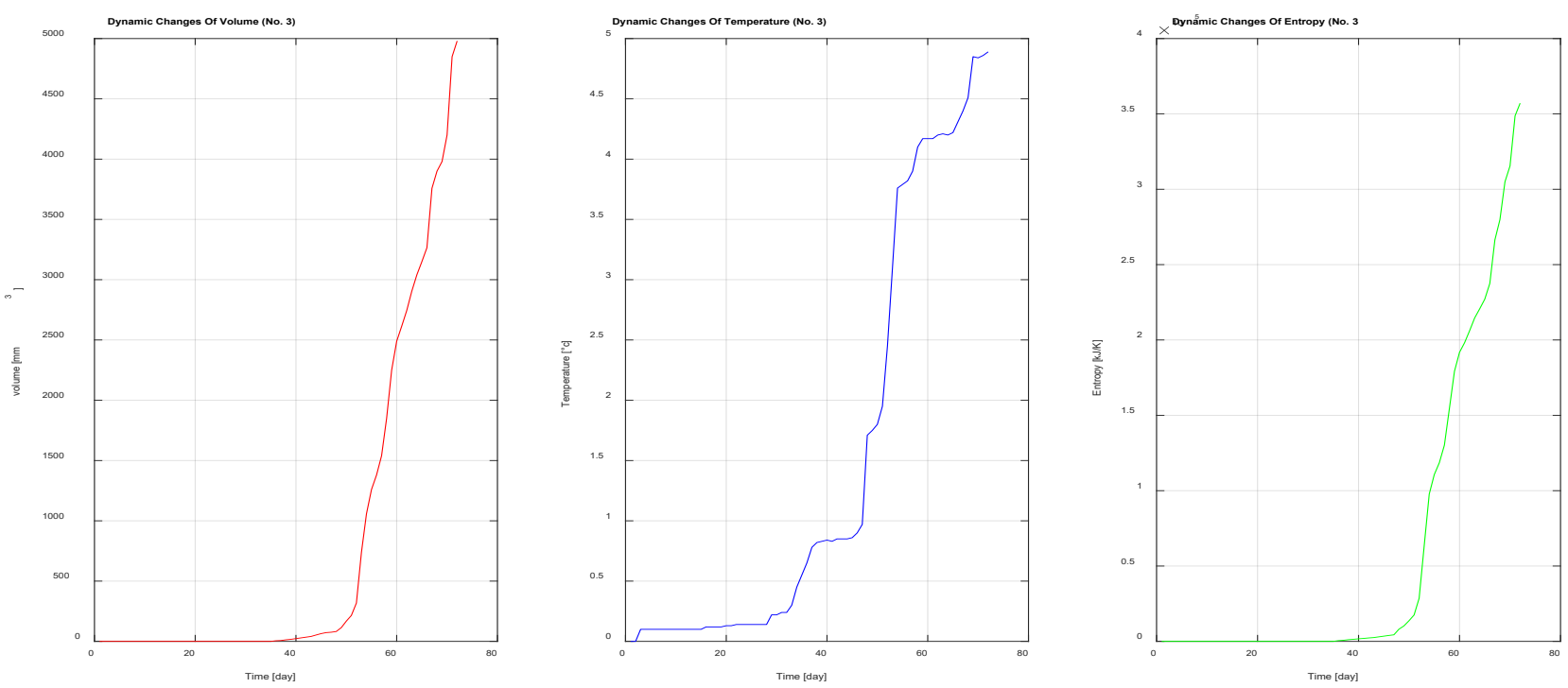

Figure 16. Dynamic changes of volume, temperature gradient, and entropy (dead mouse No.3). 

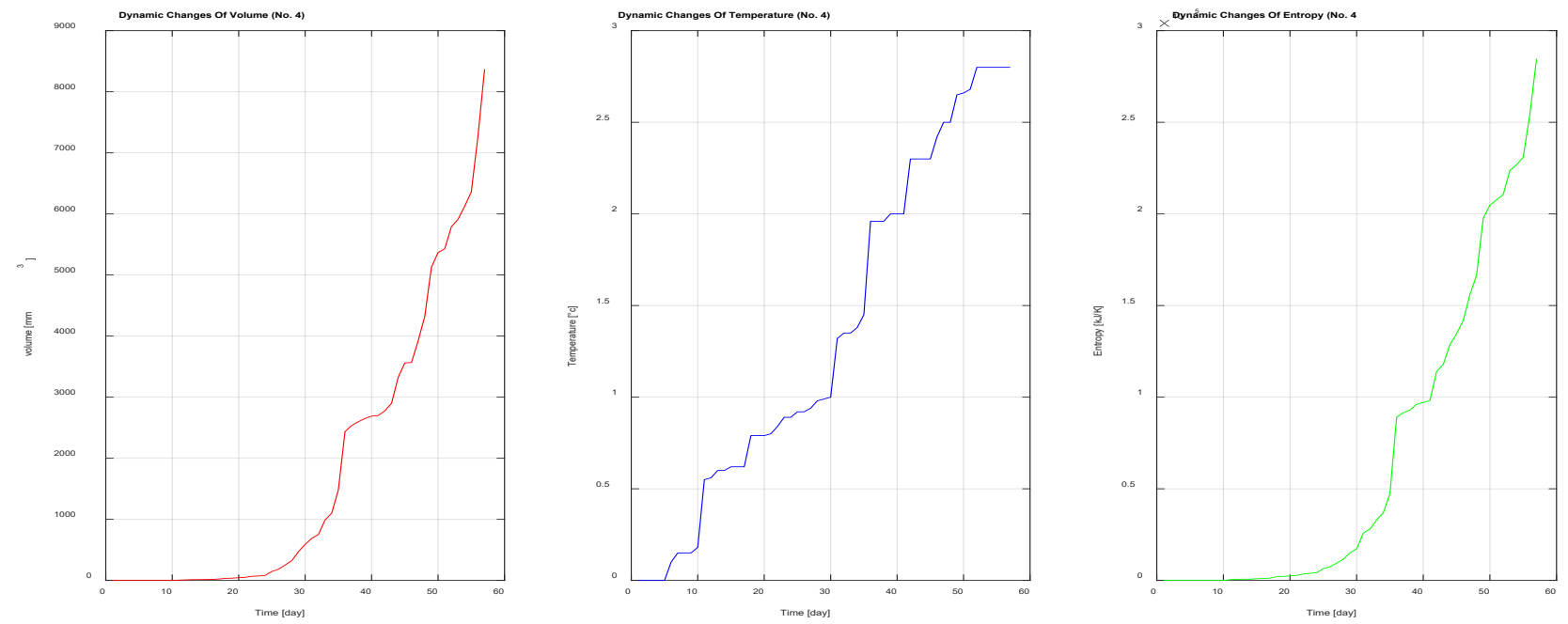

Figure 17. Dynamic changes of volume, temperature gradient, and entropy (dead mouse No.4).
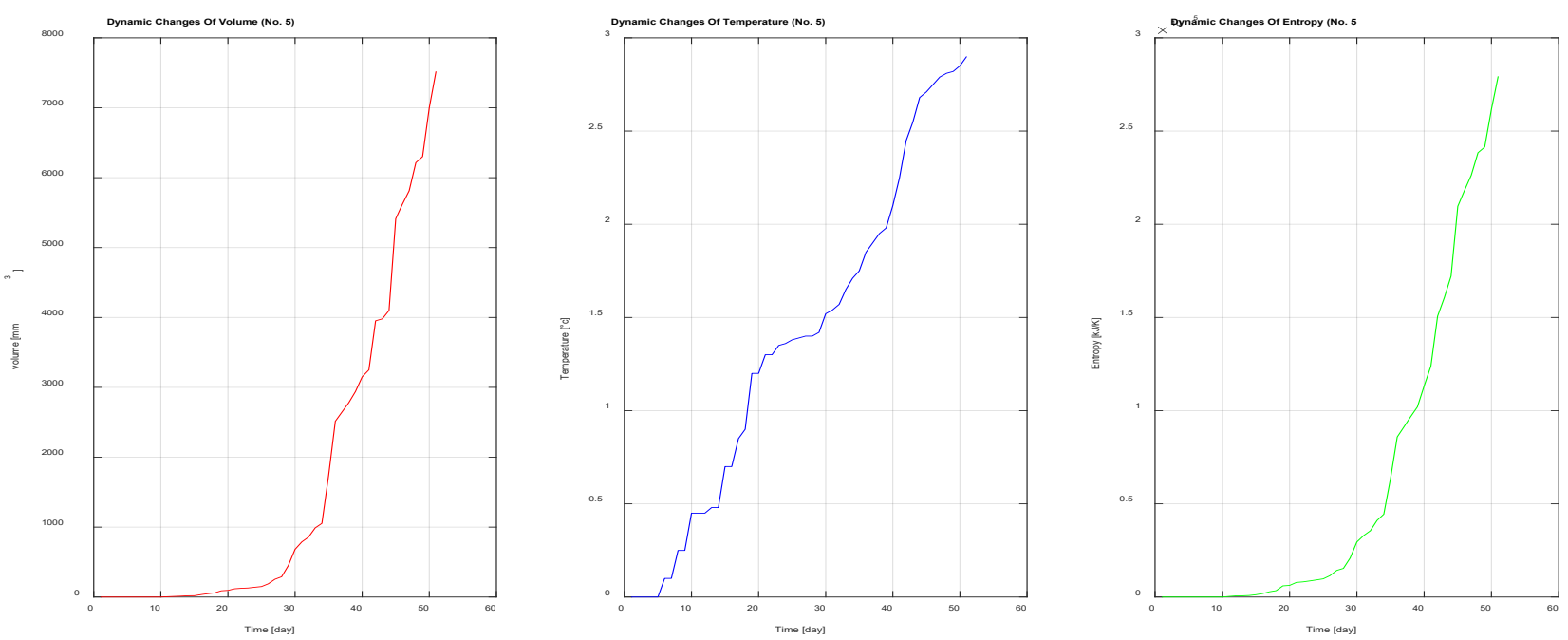

Figure 18. Dynamic changes of volume, temperature gradient, and entropy (dead mouse No.5).
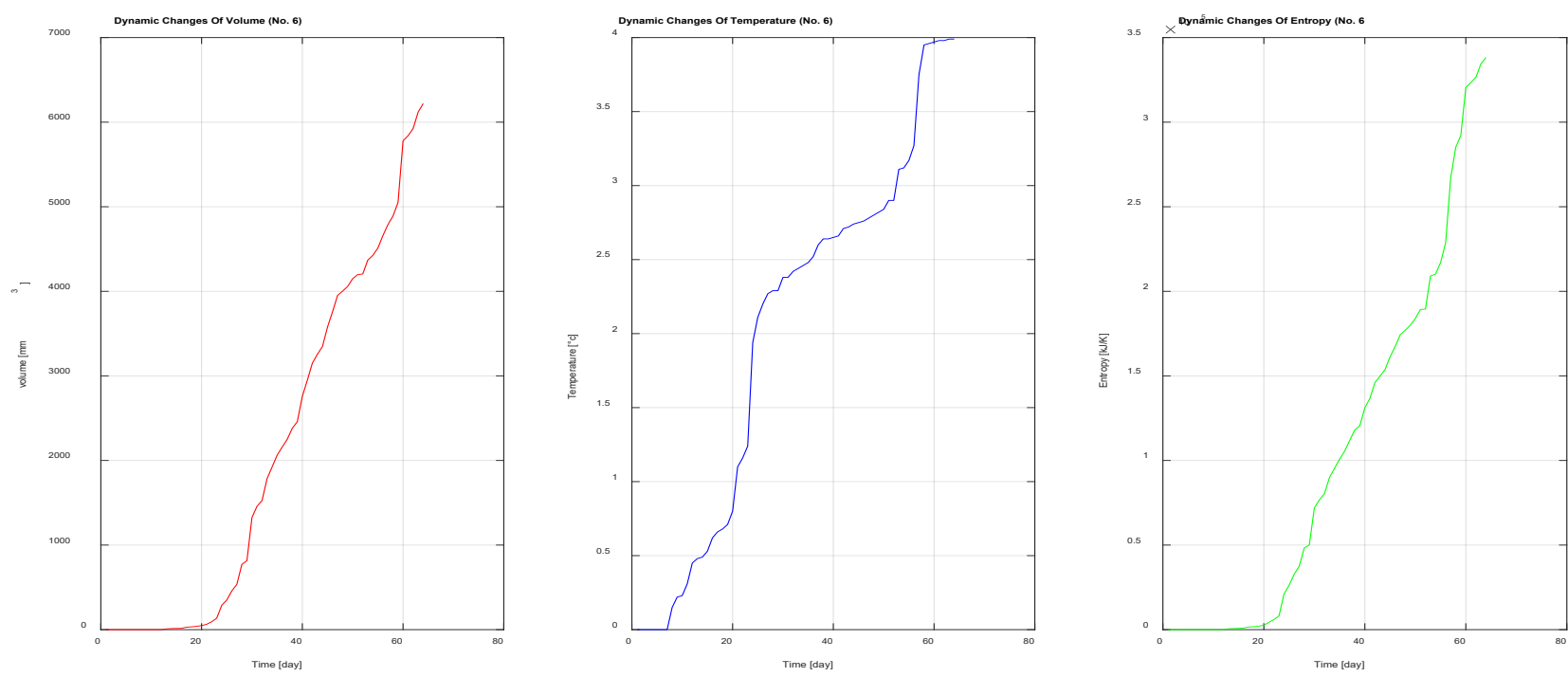

Figure 19. Dynamic changes of volume, temperature gradient, and entropy (dead mouse No.6). 
In both groups, the dynamic behavior of thermodynamic variables is the same. Based on the curves, a tumor was detected first due to the temperature changes by using thermal imaging. The maximum temperature gradient in the recovered mice group was about $1-2{ }^{\circ} \mathrm{C}$, but in the other group, it even reached $4-5^{\circ} \mathrm{C}$.

\section{Discussion and conclusions}

In this approach, we have studied and tested the changes in the volume, temperature, and entropy of the 4T1 breast tumor in $55 \mathrm{Bulb} / \mathrm{c}$ mice over time. We have measured the changes in the volume and temperature gradient of tumor cells during the stages of appearance and growth of cancer every day.

As indicated in this study's experimental results, the entropy generation of cancer changes in time due to the progression of the tumor, as mentioned in Ref. [16-18]. So, the results of this study are confirmed by Ref. [16-18].

According to the extrema entropy generation change $[16,18]$, the present study results are approved. The entropy generation during the progression of the tumor is as predicted by Luica [16].

The results of entropy generation progression in this study's tumor tissues are confirmed by the data gathered by Deisboeck, Berens, Kansal, and Torquato [17] on brain and breast tumors based on the thermodynamic engineering concept that proposed by Lucia [18].

Furthermore, it is also proof of the basic theory proposed by Norton [41] on the principle rule of the fractal geometry of the tumors. The geometry of the tumor is associated with the spatial gradient of the entropy generation $[18,25]$.

Also, the experimental results and entropy generation are agreed with the results obtained by Lucia [16].

Results obtained by the thermography of temperature changes in tumor tissue are consistent with Agnelli et al. investigation [42].

According to the results, as the tumor volume increases, the temperature gradient increases too. The temperature changes can be detected before the appearance of a volumetric tumor by using thermal imaging, so thermal imaging can be used as a widely available, non-invasive, quick, accurate, and objective measurement tool for detecting cancer.

By using a proven relation, we have calculated the entropy values over time. The entropy values can be used as a powerful tool for measuring cancer cells' invasion, progression, and robustness.

By using the obtained data, we can model the dynamic behavior of cancer as a thermodynamic system. This model can be applied in the prediction of different stages of cancer and its stationary states. We can calculate the critical value of the tumor volume and predict the time of metastasis. We hope it can be used for anticancer therapy plans.

In future studies, classical models and artificial neural networks can achieve a dynamic model of the tumor progression using experimental data. Also, the metastatic position and time can be predicted by the transient model of the tumoral tissue by using entropy generation values.

\section{Acknowledgments}

The authors would like to thanks and acknowledge Tehran University of Medical Sciences Preclinical Core Facility (TPCF), Tehran, Iran, for supporting and providing the in vivo Micro PET imaging and image processing services for this research.
Also, the author acknowledges the Laboratory of the Medical Immunology of Tehran University of Medical Sciences for all the scientific supports during the experimental investigation.

\section{References:}

[1] World Health Organization 2018 [cited 2019 June]; Available from http://www.who.int/es/.

[2] Song, C., et al., Thermographic assessment of tumor growth in mouse xenografts. International journal of cancer, 2007. 121(5): p. 1055-1058.

[3] Head, J.F., et al., The important role of infrared imaging in breast cancer. IEEE Engineering in Medicine and Biology Magazine, 2000. 19(3): p. 52-57.

[4] Jiang, L., et al., A perspective on medical infrared imaging. Journal of medical engineering \& technology, 2005. 29(6): p. 257-267.

[5] Jones, B.F., A reappraisal of the use of infrared thermal image analysis in medicine. IEEE transactions on medical imaging, 1998. 17(6): p. 1019-1027.

[6] Keyserlingk, J.R., et al., Functional infrared imaging of the breast. IEEE Engineering in Medicine and Biology Magazine, 2000. 19(3): p. 30-41.

[7] Wallace, J.D. and G.D. Dodd, Thermography in the diagnosis of breast cancer. Radiology, 1968. 91(4): p. 679-685.

[8] Ma, M., Zhang, Y., \& Gu, N. (2018). Estimation the tumor temperature in magnetic nanoparticle hyperthermia by infrared thermography: Phantom and numerical studies. Journal of Thermal Biology, 76, 8994. doi:https://doi.org/10.1016/j.jtherbio.2018.07.004

[9] Kennedy, D.A., T. Lee, and D. Seely, A comparative review of thermography as a breast cancer screening technique. Integrative cancer therapies, 2009. 8(1): p. 916.

[10] Jo, J., \& Kim, H. (2016). Comparison of abdominal skin temperature between fertile and infertile women by infrared thermography: A diagnostic approach. Journal of Thermal Biology, 61, 133-139. doi:https://doi.org/10.1016/j.jtherbio.2016.09.009

[11] Uchida, I., Y.O.Y. Ohashi, and T. TorajiIrifune, 乳房 寸一干夕元 $A D$ 二/比一一夕\{二上石定量骸断.

[12] Kaur, S.D., The Complete Natural Medicine Guide to Breast Cancer: A Practical Manual for Understanding, Prevention \& Care. 2003: Robert Rose Incorporated.

[13] Das, K., \& Mishra, S. C. (2015). Simultaneous estimation of size, radial and angular locations of a malignant tumor in a 3-D human breast - A numerical study. Journal of Thermal Biology, 52, 147-156. doi:https://doi.org/10.1016/j.jtherbio.2015.07.001

[14] H. M. Patil and R. Maniyeri, "Finite difference method based analysis of bio-heat transfer in human breast cyst," Therm. Sci. Eng. Prog., vol. 10, pp. 42-47, 2019, doi: https://doi.org/10.1016/j.tsep.2019.01.009.

[15] M. Etehadtavakol and E. Y. K. Ng, "Survey of numerical bioheat transfer modelling for accurate skin surface measurements," Therm. Sci. Eng. Prog., vol. 20, 
p. 100681, 2020, https://doi.org/10.1016/j.tsep.2020.100681.

[16] Lucia, U., Entropy generation and cell growth with comments for a thermodynamic anticancer approach. Physica A: Statistical Mechanics and its Applications, 2014. 406: p. 107-118.

[17] T.S. Deisboeck, M.E. Berens, A.R. Kansal, S. Torquato, A.O. Stemmer-Rachamimov, E.A. Chiocca, Pattern of self-organization in tumour systems: Complex growth dynamic in a novel brain tumour spheroid model. Vol. 34. 2001. 115-34.

[18] Lucia, U., Thermodynamics and Cancer stationary states. Physica A: Statistical Mechanics and its Applications, 2013. 392(17): p. 3648-3653.

[19] Luo, L., Entropy production in a cell and reversal of entropy flow as an anticancer therapy. Vol. 4. 2009. 122-136.

[20] T.Karalis, K.T. Karalis, K.N .Papavasileioy; "GROWTH OF MALIGNANT CELLS AND THERMODYNAMICS", Journal of Mechanics in Medicine and Biology, 16(02): p. 1650006 (2016).

[21] Kitano H (2007) Towards a theory of biological robustness. Mol Syst Biol 3: 137.

[22] Rockmore R (2005) Cancer complex nature. Santa Fe Institute Bulletin 20: 18-21.

[23] Lucia, U., Mathematical Consequences of Gyarmati Principle in Rational Thermodynamics. Vol. 110. 1995. 1227-1235.

[24] Lucia, U. Probability, ergodicity, irreversibility and dynamical systems. in Proceedings of the Royal Society of London A: Mathematical, Physical and Engineering Sciences. 2008. The Royal Society.

[25] Lucia, U., Irreversibility in biophysical and biochemical engineering. Physica A: Statistical Mechanics and its Applications, 2012. 391(23): p. 59976007.

[26] Lucia, U., Maximum or minimum entropy generation for open systems? Vol. 391. 2012. 3392-3398.

[27] U. Lucia, Stationary open systems: a brief review on contemporary theories irreversibility, Physica A 392( (5) (2013) 1051-1062.

[28] A. Bejan, Advance Engineering Thermodynamics, John Wiley, NJ, 2006.

[29] E. Schr"odinger, What is Life? Physical Aspects of Living Cell, Cambridge: Cambridge University Press, 1948
[30] Luo, L., Entropy production in a cell and reversal of entropy flow as an anticancer therapy. Vol. 4. 2009. 122-136.

[31] E.Izquierdo-Kulich, E. Alonso-Becerra, and J.M. Nieto-Villar; " Entropy production rate for avascular tumor growth",Journal of Modern Physics, 2(06): p. 61 (2011)

[32] Breitkreutz, D., et al., Molecular signaling network complexity is correlated with cancer patient survivability. Proceedings of the National Academy of Sciences, 2012. 109(23): p. 9209-9212.

[33] Wayman, J. A. \& Varner, J. D. Biological systems modeling of metabolic and signaling networks. Curr. Op. Chem. Eng. 2, 1-8 (2013).

[34] Kawahara, M. \& Nagamune, T. Engineering of mammalian cell membrane proteins. Curr. Op. Chem. Eng. 1, 411-417 (2012).

[35] Nicolis, G. and I. Prigogine, Self-organization in nonequilibrium systems. Vol. 191977. 1977: Wiley, New York.

[36] P, G. and P. I, Thermodyamic Theory of Structure, Stability and Fluctuations. 1973.

[37] Pulaski, B.A. and S. Ostrand-Rosenberg, Mouse 4T1 breast tumor model. Current protocols in immunology, 2000. 39(1): p. 20.2. 1-20.2. 16.

[38] Xie, W., et al., evaluation of the ability of digital infrared imaging to detect vascular changes in experimental animal tumours. International journal of cancer, 2004. 108(5): p. 790-794.

[39] Zhang, M.-Y., et al., Micro-positron emission tomography imaging of angiogenesis based on $18 \mathrm{~F}$ RGD for assessing liver metastasis of colorectal cancer. Hepatobiliary \& Pancreatic Diseases International, 2021.

[40] Yu, W., et al., 18F-HX4/18F-FMISO-based micro PET for imaging of tumor hypoxia and radiotherapyassociated changes in mice. Biomedicine \& Pharmacotherapy, 2019. 119: p. 109454.

[41] Norton L. Conceptual and practical implications of breast tissue geometry: toward a more effective, less toxic therapy. Oncologist. 2005 Jun-Jul;10(6):370-81. doi: 10.1634/theoncologist.10-6-370. PMID: 15967831.

[42] Agnelli, J.P., A.A. Barrea, and C.V. Turner, Tumor location and parameter estimation by thermography. Mathematical and Computer Modelling, 2011. 53(7): p. 1527-1534. 